\title{
Adiabatic pumping of two-dimensional electrons in a ratchet-type lateral superlattice
}

\author{
E. M. Höhberger ${ }^{\text {a) }}$ and A. Lorke ${ }^{\text {b) }}$ \\ Sektion Physik and Center for Nanoscience, LMU, D-80539 München, Germany \\ W. Wegscheider ${ }^{\mathrm{c}}$ and M. Bichler \\ Walter-Schottky-Institut, TUM, D-85747 Garching, Germany
}

(Received 28 April 2000; accepted for publication 22 January 2001)

\begin{abstract}
A pair of interdigitated gates, interlacing off center, is used to generate a widely tunable one-dimensional lateral superlattice in the two-dimensional electron gas at a GaAs/AlGaAs heterointerface. Magnetotransport measurements with statically biased gates demonstrate that the conduction-band modulation is strongly influenced by higher harmonics, thus allowing the realization of both symmetric and ratchet-like periodic potential modulations. Temporally phase-shifted ac biasing of the gates results in adiabatic pumping of electrons. The generated dc current increases linearly with the pumping frequency and can be reversed in situ by inverting the phase shift. (C) 2001 American Institute of Physics. [DOI: 10.1063/1.1355672]
\end{abstract}

Pumping is characterized by the occurrence of a net flux of particles in response to a periodic potential perturbation of an externally unbiased system. Although this mechanism is well known and peristaltic pumps are technologically exploited in many fields, its applications are still a fruitful field for science. In recent years, adiabatic electron pumps have received considerable experimental ${ }^{1-6}$ and theoretical ${ }^{7-9}$ attention. Realizations reported range from single ${ }^{1,2}$ or coupled $^{3}$ quantum dots driven in either the Coulomb blockade ${ }^{2,3}$ or the open regime, ${ }^{1}$ as well as surface acoustic wave devices ${ }^{4,5}$ to devices based on time-dependent lateral superlattices used in commercial charge-coupled-device (CCD) cameras. ${ }^{6}$ Following the fundamental proposition by Thouless, ${ }^{7}$ a theoretical understanding of many kinds of parametric pumps has been developed. ${ }^{8,9}$

All of these electron pumps are based on an adiabatic, cyclic ac deformation of the confining potential resulting in a dc current through the structure. This requires at least two spatially separated, independently addressable sets of parameters which change cyclically and sufficiently slow in time with a nonzero temporal phase shift. For a nonperiodical system like a quantum dot, these parameters correspond to two single barriers, e.g., two gates controlling the charge on the dot. In spatially periodic pumping devices one has to be slightly more cautious by ensuring that the parameters change in a clearly distinguishable sequence. In this case, the two parameters can be conceived as two standing waves adding up to a traveling wave in which the carriers are transported. If the spatial shift between the two parameters equals $\pi$, there is no distinguished direction, in terms of the wave picture, the waves extinguish each other. For this reason many commercial CCD cameras are based on three-phase geometries.

In this letter, we report the peristaltic pumping of ballis-

\footnotetext{
${ }^{a}$ Electronic mail: Eva.Hoehberger@physik.uni-muenchen.de

${ }^{b)}$ Present address: Solid State Physics Laboratory, Gerhard-MercatorUniversität, D-47048 Duisburg, Germany.

${ }^{c}$ Present address: Institut für Angewandte und Experimentelle Physik, Universität Regensburg, D-93040 Regensburg, Germany.
}

tic electrons in a two-dimensional electron gas (2DEG) using only two fluctuating potential barriers. We show that each of the driving potentials may be periodic in time and space. However, both a spatial and a temporal phase shift between the input signals is required for a net flux of electrons. More generally, the system can be interpreted as a ratchet: ${ }^{10} \mathrm{~A}$ net flux of particles occurs due to broken spatial inversion symmetry in a system subjected to time-correlated fluctuations, ${ }^{11}$ which can be induced by either colored noise, a fluctuating force, or, like in our case, a fluctuating potential.

Two samples, A and B, were prepared from modulationdoped GaAs/AlGaAs heterostructures with the 2DEG located $37 \mathrm{~nm}$ beneath the surface. A Hall geometry was defined using optical lithography and wet-chemical etching. Ohmic contacts were provided by evaporated AuGeNi pads alloyed at $420^{\circ} \mathrm{C}$. Two interdigitated gates which interlace off center as shown in Figs. 1(b) and 1(c), were defined on the Hall bar using electron-beam lithography and evaporation of $15 \mathrm{~nm} \mathrm{NiCr}$. As determined by atomic-force microscopy, the resulting one-dimensional lateral superlattice of sample A exhibits 95 periods with a length $a$ of $775 \mathrm{~nm}$. The

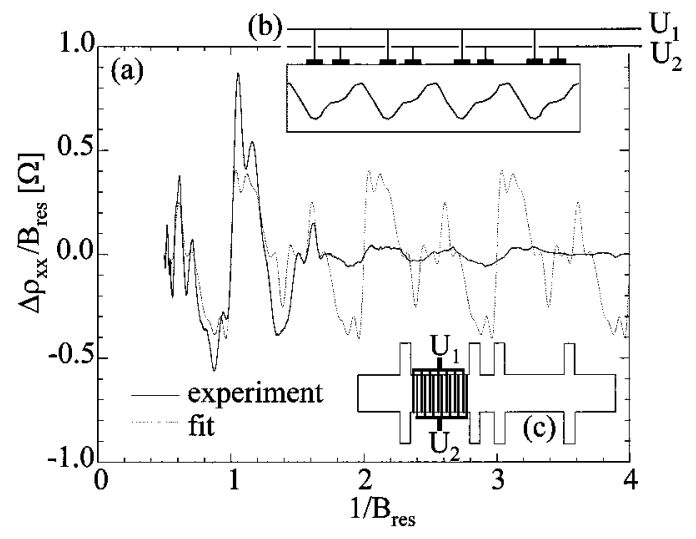

FIG. 1. (a) Oscillatory part of the magnetoresistance of sample A for a ratchet potential $U_{1}=-100 \mathrm{mV}+U_{0}, U_{2}=-50 \mathrm{mV}+U_{0}$ with offset $U_{0}=+150 \mathrm{mV}$. The dotted line is the result of a Fourier analyis. (b) Schematic of the applied potential modulation. (c) Hall bar and off-centered interdigitated gate geometry used in the experiment. 
width of a single finger $w$ is $256 \mathrm{~nm}$ and the spacings $s_{1}$ and $s_{2}$ between two adjacent fingers are 188 and $75 \mathrm{~nm}$, respectively. Sample B contains a superlattice of the same overall lateral size but with 74 periods and dimensions $a$ $=1000 \mathrm{~nm}, w=200 \mathrm{~nm}, s_{1}=400 \mathrm{~nm}$, and $s_{2}=200 \mathrm{~nm}$.

Biasing the gates with two independent voltages allows the generation of a wide range of periodic potentials modulating the conduction band. In particular, the off-centered setup of the interdigitated gate electrodes allows the definition of both inversion symmetric and ratchet-shaped lateral superlattices. Furthermore, a given potential modulation can be switched off or inverted in situ by exchanging the voltages applied to the gates so that spurious effects like, e.g., thermal voltages, can readily be eliminated from measurements.

Commensurability oscillations ${ }^{12,13}$ in the low-field magnetoresistance of a 2DEG reflect ballistic transport in the lateral superlattice and can be employed to map out the exact shape of the potential modulation. ${ }^{14}$ In the following, we use commensurability oscillations to determine whether a given potential modulation with distinct symmetry properties can be transferred into the 2DEG with sufficient accuracy. A quasiclassical ballistic model to describe commensurability oscillations was developed by Beenakker ${ }^{15}$ and generalized to include higher harmonics by Gerhardts. ${ }^{16}$ For a given weak potential modulation of the 2DEG in depth $d, V(x, z$ $=d)=\sum_{n=-\infty}^{+\infty} V_{n, z=d} \exp (2 \pi n i x / a)$ with $V_{n, z=d}=V_{-n, z=d}^{*}$, the oscillating contribution $\Delta \rho_{x x}$ to the low-field magnetoresistivity $\rho_{x x}$ is given by

$$
\frac{\Delta \rho_{x x}}{\rho_{0}}=\frac{2}{E_{F}^{2}}\left(\frac{l_{f}}{a}\right)^{2} B_{\text {res }} \sum_{n=-\infty}^{+\infty} n\left|V_{n, z=d}\right|^{2} \sin \left(2 \pi n \frac{1}{B_{\text {res }}}\right),
$$

with the zero-field resistivity $\rho_{0}$, Fermi energy $E_{F}$, and the electron mean-free path $l_{f}=\tau v_{F}$. The rescaled magnetic field $B_{\text {res }}$ can be expressed in terms of the superlattice period $a$ and the classical cyclotron radius $R_{c}$ as $B_{\text {res }}=a / 2 R_{c}$.

From Eq. (1a) it can be seen that the low-field magnetoresistivity displays an oscillatory structure which is characterized by the absolute squares of the Fourier coefficients $V_{n, z=d}$ and repeated periodically at $B_{\text {res }}^{-1}=1,2,3, \ldots$ As $\Delta \rho_{x x}$ is given in terms of $\left|V_{n, z=d}\right|$ and thus contains no phase information, the exact shape of the potential cannot be deduced without further assumptions on the symmetry of the potential. However, the existence of higher harmonics at the location of the 2DEG, which is essential for symmetry breaking potentials, can still be verified. Figure 1(a) shows commensurability oscillations in the four-terminal magnetoresistance across the superlattice, $\Delta \rho_{x x}$, plotted against $1 / B_{\text {res }}$. The displayed data were measured at $4.2 \mathrm{~K}$ in a standard Hall bar geometry in sample A. The applied gate voltages were $U_{1}=-100 \mathrm{mV}+U_{0}$ and $U_{2}=-50 \mathrm{mV}+U_{0}$. The offset $U_{0}=+150 \mathrm{mV}$ was used to eliminate the built-in modulation of the conduction band caused by the Schottky contact between the gates and the semiconductor surface. The carrier density was determined from Shubnikov-de Haas oscillations to be $n_{s}=6.44 \times 10^{11} \mathrm{~cm}^{-2}$. For the given gate voltages, three periods were resolved (solid line) and fitted with a seventh-order Fourier series [dotted line in Fig. 1(a)]. The existence of higher harmonics is reflected by a characteristic double-peak structure in the commensurability

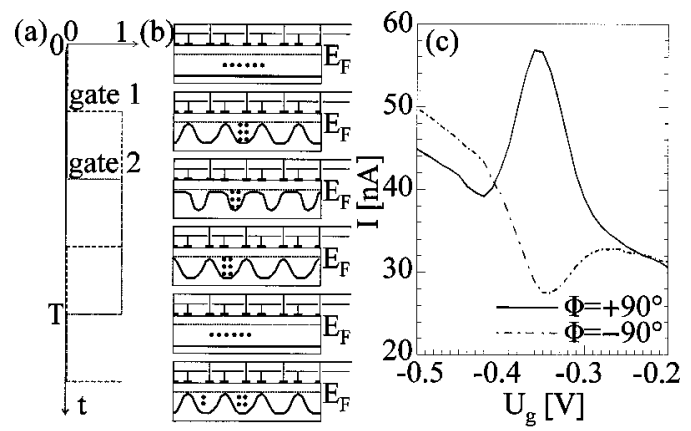

FIG. 2. (a) Temporal sequence of the rectangular gate pulses used to pump electrons. The passive state of a gate is denoted 0 , the active state 1 . Gate 1 is depicted by a dashed line, gate 2 by a dotted line. (b) Schematic of the pumping cycle for case 1 . The solid line displays the conduction-band modulation of the 2DEG. The dots symbolize the 2D electrons and demonstrate that the pumping efficiency after one cycle is $P_{1}=1 / 3$. (c) Pumped current through sample B for $f=10 \mathrm{kHz}$. The solid line represents the case of positive, the dash-dotted line the case of negative phase $\phi$ between the gates.

oscillations. Equation (1a) shows that this can only be the case if the 2DEG is under the influence of more than the first harmonic of the applied potential. As long as at least two Fourier components are present, the off-center symmetry of the interdigitated gates is transfered into the 2DEG. Numerical calculations based on an image charge model including Thomas-Fermi screening also showed a reflection of the shape of the applied potential in the resulting potential modulation. ${ }^{17}$ The given gate geometry can thus be employed to generate both symmetric and ratchet-type potential modulations.

In order to pump electrons through the structure, the applied gate voltages were adiabatically changed following a periodic cycle described in Figs. 2(a) and 2(b). Using a standard programmable pulse generator, each gate was biased with a rectangular voltage pulse with amplitude $U=U_{g}$ $+U_{0}$ and frequency $f$. The absolute value of the temporal phase shift between the gates was $|\phi|=90^{\circ}$. Hence, one pumping cycle consists of four steps, as depicted in Fig. 2(a): In the first step, none of the gates is activated (state 0 ). In the next step, one of the gates is switched on (state 1). Then, both gates are in the active state 1 . In the last step, the first gate is switched off to state 0 again so that only the second gate remains in state 1 . A similar pumping cycle was described theoretically by Parrondo. ${ }^{18}$ In our case, the activated state of a gate is characterized by a strongly negative bias resulting in the depletion of the 2DEG. The passive state can either just compensate the offset $U_{0}$ [case 1, c.f. Fig. 2(b)] or consist in a positive applied voltage (case 2, not illustrated). To keep the model simple, the shape of the potential modulation is approximated by the externally applied rectangular pulses assuming $w=0.4 a, s_{1}=0.2 a$, and $s_{2}=0$. These assumptions are justified by the analysis of the static potential modulations described above: In the strongly modulated case, a comparison between the measured commensurability oscillations and theoretical simulations ${ }^{17}$ revealed that the potential modulation in the 2DEG, $d=37 \mathrm{~nm}$ below the sample surface, broadens by a factor of almost 2 so that the smaller spacing $s_{2}$ between the gates can hardly be resolved at $z=d$.

The resulting pumping current can be calculated by the 
product of the fraction $P$ of the electrons being pumped, the total number of electrons in the structured area $N_{s}$, the electron charge $e$ and the pumping frequency $f . N_{s}$ is given by the carrier density $n_{s}$ times the structured area $F=74 \mu \mathrm{m}$ $\times 40 \mu \mathrm{m}$, so that

$$
I_{i}=P_{i} \cdot n_{s} \cdot F \cdot e \cdot f \text {. }
$$

This is illustrated for case 1 in Fig. 2(b), where the electrons of the 2DEG are depicted as small dots. From Fig. 2(b) it seems obvious that within one pumping cycle one third of the carriers is pumped forward $\left(P_{1}=1 / 3\right)$. This simple picture ignores the buildup of charge in the depleted region underneath the superlattice which takes place for continuous pumping. A careful evaluation shows that this effect can be described in terms of a geometric series. Hence, we end up with an effective fraction of pumped particles of $P_{1}=1 / 2$. Similarly, it can be shown that for case 2 all electrons are pumped and $P_{2}=1$.

The current flowing under pumping conditions was measured from source to drain for $\phi= \pm 90^{\circ}$ at $4.2 \mathrm{~K}$ for different pumping amplitudes $U_{g}$ and frequencies $f$. Figure 2(c) shows the current through sample B for $f=10 \mathrm{kHz}$ as a function of amplitude $U_{g}$. The carrier density of the static sample was $n_{s}=4.70 \cdot 10^{11} \mathrm{~cm}^{-2}$. The zero of both curves at $U_{g}=0$ was shifted by $32 \mathrm{nA}$ due to thermal currents in the sample. For small amplitudes $U_{g}$ pumping is not possible since the modulation is far below the Fermi energy. This allows for a backflow of displaced electrons corresponding to a "leaky" pump. For larger $U_{g}$ a current was measured, the sign of which depends on the direction of the phase shift. The absolute value of the current reaches its maximum value when the pumping amplitude equals the depletion voltage of the 2DEG known from $C-V$ measurements. For even larger amplitudes, the pumped current decreases again. In this regime the center part of the superlattice loses contact with the source and drain regions and floats with respect to the 2DEG as the electron channel is strongly depleted under the active gate. The linear background seen in Fig. 2(c) could be traced back to the rectification of capacitive currents arising in nonideal AuGe contacts. In order to eliminate such parasitic effects the curves for forward and backward pumping with $\phi$ $= \pm 90^{\circ}$, respectively, were subtracted from each other. The net pumping current is then given by half the value of this difference and again reaches its maximal value at the depletion voltage $U=-0.35 \mathrm{~V}+U_{0}$. In Fig. 3(a), the maximal pumping current is plotted with respect to the pumping frequency $f$ for both cases 1 and 2. The curves display linear behavior for values below $f=180 \mathrm{kHz}$ and saturate at higher frequencies. The saturation frequency is in good agreement with an approximated $R C$ frequency of $188 \mathrm{kHz}$ for an estimated resistance of $100 \mathrm{k} \Omega$ between the gates and the 2DEG. The observed values for the fraction of pumped particles are $P_{1}=0.44 \pm 0.04$ and $P_{2}=0.86 \pm 0.08$. Hence, the ratio of the pumping current for cases 1 and 2 equals the estimated value of $P_{1} / P_{2}=0.5$ within the accuracy of the error bars.

Figure 3(b) shows the normalized pumping current for several values of the phase $|\phi|$ between the applied gate voltages for $f=10 \mathrm{kHz}$. It reaches its maximal value for $|\phi|=90^{\circ}$. Its dependence on the phase is approximately described by a sinusoidal curve, similar to the case of an open quantum-dot pump. ${ }^{1}$

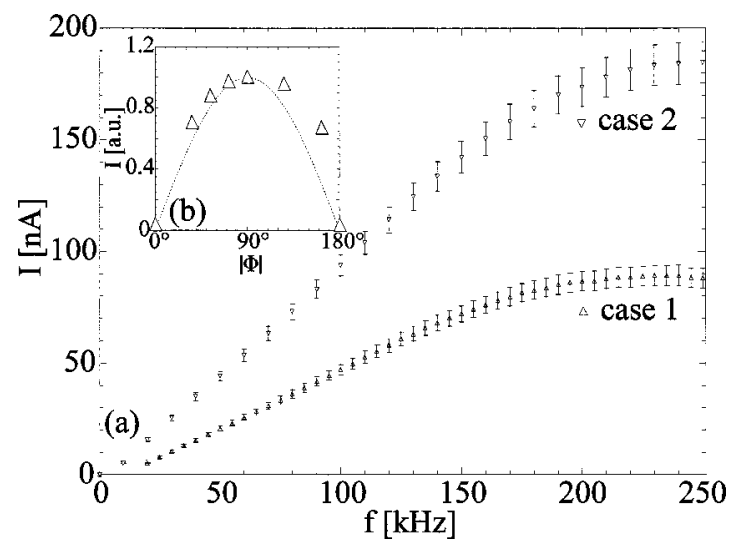

FIG. 3. (a) Maximum pumping current as a function of the pumping frequency for case $1\left(P_{1}=1 / 2\right)$ and case $2\left(P_{2}=1\right)$. The estimated $R C$ frequency is $188 \mathrm{kHz}$. (b) Normalized pumping current for different absolute values of the pumping phase $\phi$.

In conclusion, we demonstrated that an interdigitated gate structure without spatial inversion symmetry can be used to create lateral superlattices of various symmetries in the 2DEG of a GaAs/AlGaAs heterostructure. Furthermore, we showed that phase-shifted biasing of the gates with rectangular voltage pulses results in adiabatic pumping of ballistic electrons. With similar structures it should also be possible to find direct evidence for ballistic transport under pumping conditions or to reach the phase-coherent transport regime at lower temperatures. These goals will be the focus of further investigations.

The authors would like to thank J. P. Kotthaus for continuous support and stimulating discussions. This work has been funded by the BMBF via Grant No. 01BM914 and a Max-Planck research award.

${ }^{1}$ M. Switkes, C. M. Marcus, K. Campman, and A. C. Gossard, Science 283, 1905 (1999).

${ }^{2}$ L. P. Kouwenhoven, A. T. Johnson, N. C. van der Vaart, C. J. P. M. Harmans, and C. T. Foxon, Phys. Rev. Lett. 67, 1626 (1991).

${ }^{3}$ H. Pothier, P. Lafarge, C. Urbina, D. Esteve, and M. H. Devoret, Europhys. Lett. 17, 249 (1992).

${ }^{4}$ V. I. Talyanskii, J. M. Shilton, M. Pepper, C. G. Smith, C. J. B. Ford, E. H. Linfield, D. A. Ritchie, and G. A. C. Jones, Phys. Rev. B 56, 15180 (1997).

${ }^{5}$ M. Rotter, A. V. Kalameitsev, A. O. Govorov, W. Ruile, and A. Wixforth, Phys. Rev. Lett. 82, 2171 (1999).

${ }^{6}$ For a review, see e.g., W. G. Ong, Modern MOS Technology: Processes, Devices and Design (McGraw-Hill, Singapore, 1987).

${ }^{7}$ D. J. Thouless, Phys. Rev. B 27, 6083 (1983).

${ }^{8}$ P. W. Brouwer, Phys. Rev. B 58, 135 (1998).

${ }^{9}$ B. Spivak, F. Zhou, and M. T. Beal Monod, Phys. Rev. B 51, 13226 (1995).

${ }^{10}$ R. P. Feynman, R. B. Leighton, and M. Sands, The Feynman Lectures in Physics (Addison-Wesley, Reading, MA, 1966).

${ }^{11}$ For a review, see, e.g., P. Hänggi and R. Bartussek, Lect. Notes Phys. 476, 294 (1996).

${ }^{12}$ R. W. Winkler, J. P. Kotthaus, and K. Ploog, Phys. Rev. Lett. 62, 1177 (1989).

${ }^{13}$ R. R. Gerhardts, D. Weiss, and K. von Klitzing, Phys. Rev. Lett. 62, 1173 (1989).

${ }^{14}$ R. J. Luyken, A. Lorke, A. M. Song, M. Streibl, J. P. Kotthaus, C. Kadow, J. H. English, and A. C. Gossard, Appl. Phys. Lett. 73, 1110 (1998).

${ }^{15}$ C. W. J. Beenakker, Phys. Rev. Lett. 62, 2020 (1989).

${ }^{16}$ R. R. Gerhardts, Phys. Rev. B 45, 3449 (1992).

${ }^{17}$ E. M. Höhberger, diploma thesis, LMU München, 1999 (unpublished).

${ }^{18}$ J. M. R. Parrondo, Phys. Rev. E 57, 7297 (1998). 